\title{
FEMORAL GIANT CELL TUMOR IN A PATIENT WITH SURGICALLY TREATED FEMORAL CONDYLE FRACTURE: MISDIAGNOSIS OR COINCIDENCE?
}

doi: $10.2478 /$ rojost-2018-0022

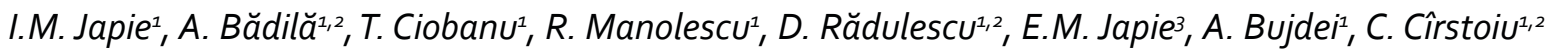 \\ ${ }^{1}$ Department of Orthopaedics and Traumatology, University Emergency Hospital, Bucharest, Romania \\ 2"Carol Davila" University of Medicine and Pharmacy, Bucharest, Romania \\ ${ }^{3}$ Department of Pediatric Orthopaedics, Maria Skłodowska Curie Children's Clinical Hospital, Bucharest, \\ Romania
}

Introduction. Giant-cell tumor of bone (GCTB) is a benign tumor with an unpredictable evolution, representing $4-5 \%$ of all primary bone tumors and $15 \%$ of benign bone tumors usually affecting 20-45 years old adults. The predilect location is the distal femur, proximal tibia, and distal radius. Case presentation. We report the case of a 31-year-old male, regardless of medical history, admitted in the emergency department (ED) for significant pain and functional impairment of the right knee, after suffering a traumatic event. Clinical examination and imaging tests established the diagnosis of lateral femoral condyle fracture.

Therefore, osteosynthesis with 4 screws was performed. Postoperative evolution was uneventful until one year later when the patient presented to the ED for pain and inflammatory aspect of the right knee, but with no history of trauma during this time. The imagistic exams of the right knee (X-ray, magnetic resonance imaging and scintigraphy) detected a tumor of the lateral femoral condyle that also affected the osteosynthesis material. Thus, the removal of screws and histopathological exam were performed, the latter establishing the diagnosis of GCTB. Taking into consideration radiological and histological aspects of the tumor and relating them to the clinical findings, the GCTB was classified in stage III Enneking. The patient underwent surgery, segmental resection of the tumor in oncological limits and arthroplasty with modular tumoral prosthesis was performed. Postoperative results at 6 and 12 months according to Musculoskeletal Tumor Society Scoring System were very good.

The key feature of this case consists of post-osteosynthesis appearance of the GCTB given the fact that only 3 cases of GCTB affecting the screw site were reported in literature.

Keywords: giant-cell tumor of bone, lateral femoral condyle fracture, modular tumoral prosthesis 